\title{
Combined SIMS-SPM Instrument for High Sensitivity and High Resolution Elemental 3D Analysis
}

\author{
T. Wirtz, ${ }^{1}$ Y. Fleming, ${ }^{1}$ U. Gysin, ${ }^{2}$ Th. Glatzel, ${ }^{2}$ E. Meyer, ${ }^{2}$ U. Maier ${ }^{3}$ and U. Wegmann ${ }^{3}$ \\ 1. Department "Science and Analysis of Materials" (SAM), Centre de Recherche Public - \\ Gabriel Lippmann, 41 rue du Brill, L-4422 Belvaux, Luxembourg \\ 2. Department of Physics, University of Basel, Klingelbergstr. 82, CH-4056 Basel, Switzerland \\ 3. Ferrovac GmbH, Thurgauerstr. 72, CH-8050 Zürich, Switzerland
}

Secondary Ion Mass Spectrometry (SIMS) is a well-established and extremely powerful technique for the chemical analysis of surfaces and thin films. Its main advantages are its excellent sensitivity, its high dynamic range, its good mass resolution and its ability to distinguish between isotopes. Due to its excellent sensitivity and thus its low detection limits, SIMS can be used to detect both major and trace elements. While SIMS was originally mainly used for depth profiling, the applications gradually shifted towards $2 \mathrm{D}$ and 3D imaging as a result of the dramatically improved spatial resolution resulting from the progress made on the instrumental side. As a consequence, new fields of application for SIMS, e.g. in life sciences and nanotechnologies, are emerging. In addition, the possibility of detecting several isotopes in parallel opens still other horizons, mainly in life sciences, where isotopic labeling is an important investigation technique.

Traditional SIMS 3D imaging is however affected by serious artifacts: while these traditional 3D reconstruction protocols and software assume that the initial sample surface is flat and the analyzed volume is cuboid, "real samples" present a surface topography, which furthermore changes during the ion bombardment as the local sputter yields depend on parameters such as the local angle of incidence of the ion beam and the crystal orientation. In addition, the situation is worsened if the sample is constituted of different materials due to preferential sputtering phenomena. As a consequence, the produced 3D images are affected by a more or less important uncertainty on the depth scale and can be distorted. Finally, significant field inhomogeneities arise from the surface topography as a result of distortion of the local electric field. These perturb both the primary beam and the trajectories of secondary ions, resulting in a number of possible artifacts, including shifts in apparent pixel position and changes in intensity.

Attempts to overcome this limitation have been done by performing ex-situ Atomic Force Microscopy (AFM) measurements on the sample surface before the SIMS analysis and on the post bombardment craters. However, the accuracy of this approach is limited. At first, the obtained AFM images only reflect the situation before or after bombardment, but give no intermediate information regarding the evolution of the roughness during sputtering. Secondly, the fact that the sample needs to be exposed to air while transferring it from the SIMS instrument to the AFM has shown to introduce considerable artifacts due to surface oxidation and surface reorganization. This also applies to samples previously analyzed with a $\mathrm{Cs}^{+}$primary ion beam, which is the case on many state-of-the-art SIMS instruments, as air exposure leads to the formation of so-called Cs dots on the sample, which again then considerably changes the surface topography of the sample. Thirdly, as (organic) SIMS measurements are frequently performed at a controlled low temperature of the sample, ex-situ AFM analysis will lead to artifacts, as the ambient conditions are different between SIMS and AFM. 
Finally, it is often very difficult to re-localize the analyzed zone, when transferring the sample between standalone SIMS and AFM instruments.

In order to obtain real high-resolution SIMS 3D analyses without being prone to the aforementioned artifacts and limitations linked to ex-situ AFM measurements, we have developed a new highprecision sample stage with an integrated SPM (Scanning Probe Microscopy) system dedicated to the Cameca NanoSIMS. With this unique tool, an in-situ combination of sequential high resolution Scanning Probe Microscopy (SPM) and high sensitivity SIMS becomes possible. In addition to being able to perform real high-sensitivity high-resolution chemical 3D imaging by recording topographical images of the sample surface in-situ before, in between and after SIMS analysis, this extremely powerful analytical tool allows combining SIMS images with valuable AFM and KPFM (Kelvin Probe Force Microscopy) data recorded in-situ in order to provide an extended picture of the sample under study.

This paper will present the prototype instrument with dedicated software, its performances and some typical examples of application.
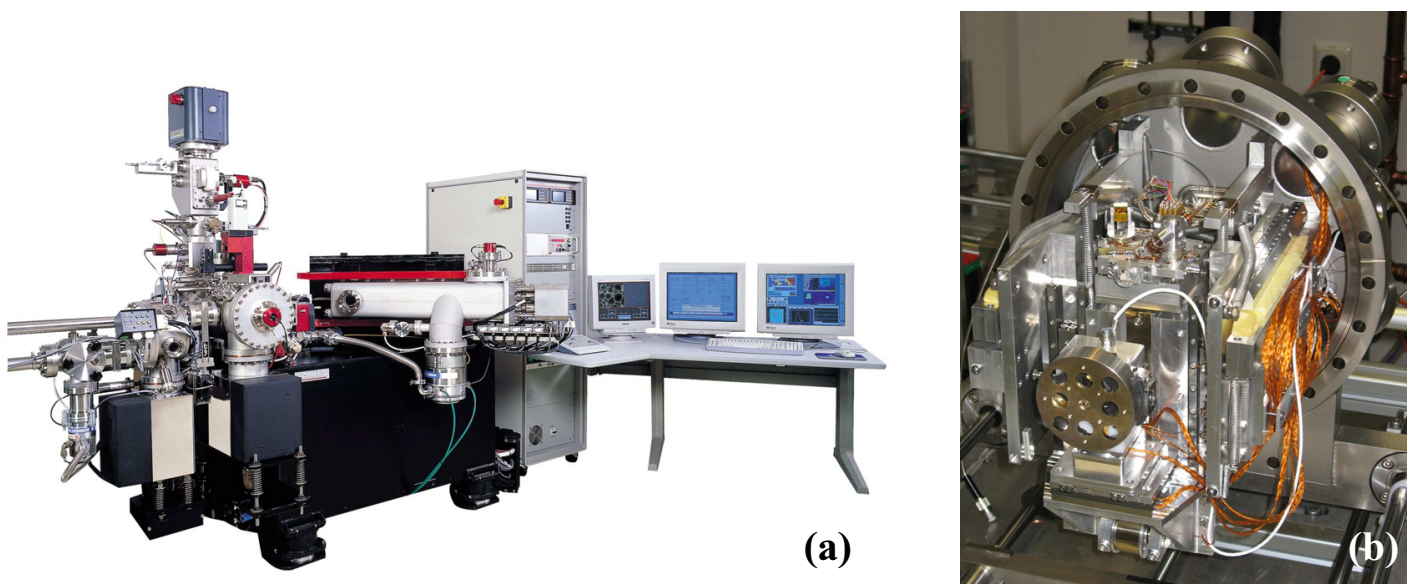

Figure 1: Photos of the prototype set-up: Cameca NanoSIMS 50 (a) and new high-precision sample stage with integrated SPM device (b).
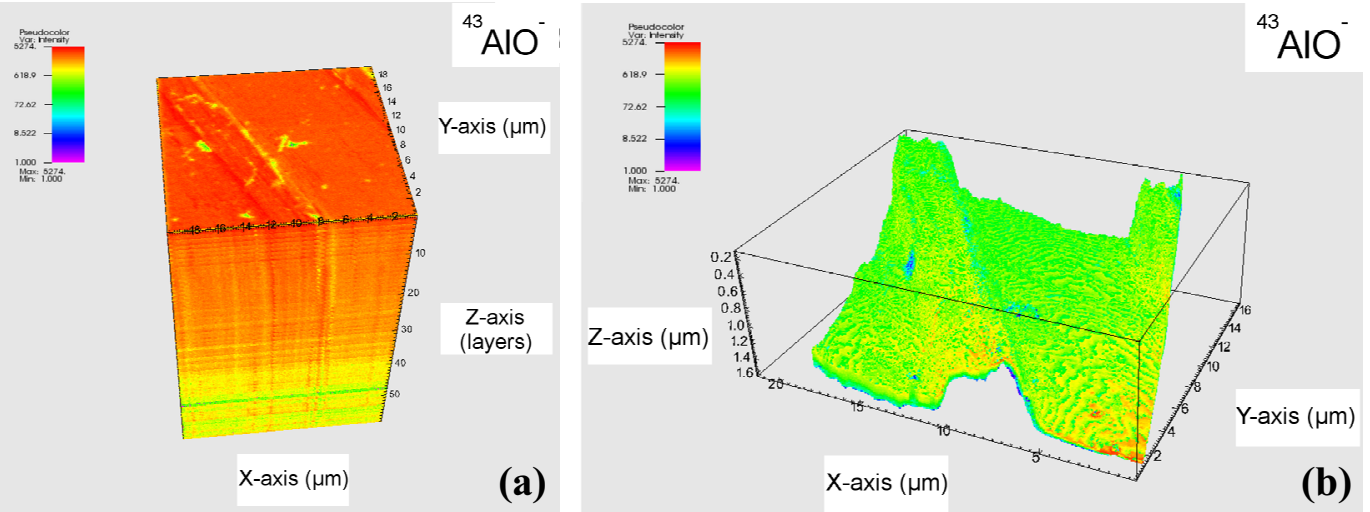

Figure 2: Al/Cu sample: (a) Traditional NanoSIMS 50 3D reconstruction of the ${ }^{43} \mathrm{AlO}^{-}$secondary ion signal. Field of view: 20x20 $\mathrm{mm}^{2}$. (b) Combined SIMS-SPM 3D reconstruction of the ${ }^{43} \mathrm{AlO}^{-}$signal. Field of view: $22.3 \times 17.3 \mu \mathrm{m}^{2}$. 